\title{
A unified European approach to high temperature defect assessment code and its incorporation in a Knowledge Base System
}

\author{
K. Nikbin \\ Mechanical Engineering Department \\ Imperial college \\ London SW7 2BX
}

\begin{abstract}
The background to high temperature defect assessment methodologies is investigated and similarities and differences are highlighted. It is clear that Europe is a leader in this developing field. Comparison of the existing European high temperature life assessment codes [1-9] lead to the conclusion that crack initiation is dealt with in different ways by the codes whereas creep and creep/fatigue crack growth are considered using the same models for analysis. At the same time whilst increasing sophistication is implemented in the analysis at the deterministic level insufficient emphasis is placed on the effects of the sensitivity and applicable bounds of the various parameters and material properties. The survey showed that for the same methods of analysis, widely different results can be derived depending on the choice of material properties, method for calculating $K$, reference stress $\sigma_{r e f}$ and $C^{*}$. The reason for the differences and the sensitivity to them is highlighted in this paper. The resulting conclusions suggest a methodology based on an expert system using both deterministic and probabilistic techniques would be beneficial. An outline of a defect assessment code structure, taking account of the major European codes, is presented which allows flexibility in terms of input of material data as well as the models used. The structure and the further development of the HIDA Knowledge Based System (KBS) is based on the concepts presented.
\end{abstract}

Keyword: life assessment, creep, fatigue, power generation, knowledge based system

\section{Introduction}

Components in the power generation and petro-chemical industry operating at high temperatures are almost invariably submitted to static and/or combined loading. They may fail by crack growth, net section rupture or a combination of both. The present paper considers the defect assessment regime of failure. Figure 1 shows a schematic diagram of the range of crack initiation and growth bahaviour that a cracked component might show. A number of defect assessment procedures (e.g. the British Energy R5 and the French A16, BS-7910 [1-6] and the German FBH and two-criteria method) [7-8] based on experimental and analytical models are available to assess crack initiation and growth and to determine the remaining useful life of such components.

It is clear from these assessment methods that the correct evaluation of the relevant fracture mechanics parameters, upon which the lifetime prediction times are dependent, is extremely important. It is also evident that the detailed calculation steps, which are proposed in these documents, do not in themselves improve the accuracy of the life prediction results. In any event as these procedures have been validated for limited sets of geometries and material data their use in other operating conditions will need careful judgement.

\section{Comparison of the present codes}

The development of codes in different countries has moved in similar directions and in many cases the methodology has been borrowed from a previously available code in another country. The early approaches to high temperature life assessment show methodologies that were based on defect-free 
assessment codes. For example ASME Code Case N-47 [9] and the French RCC-MR [10], which have many similarities, are based on lifetime assessment of uncracked structures. More recent methods make life assessments based on the presence of defects in the component. For example the German flat bottom hole approach $(\mathrm{FBH})$ considers crack detection and characterisation and the German two criteria method [7,8] regards initiation as the important factor in life assessment and does not deal with the crack growth regime. The more advanced codes dealing with defects over the range of creep and creep/fatigue interaction in initiation and growth of defects are the British Energy R5 [3], the French A16 [4,5] and BS-7910 [6] which have clear similarities in terms of methodology.

The two British high temperature codes BS-7910 and R5 attempt to deal comprehensively with assessment and remaining life estimation procedures that can be used at the design stage and for in service situations. They stress a life assessment approach that allows the expert to decide upon the applicability of the predictions in relation to the operating circumstances. The concept implies that the codes need to show that they are both reliable and understandable over a range of material and loading conditions that may not been have previously examined or validated by the code developer. This is particularly important as new higher strength steels, for which little or no long-term material properties data are available, are developed or used by the power industry.

Therefore the trend in the development of the codes is suggesting that increased flexibility in dealing with the information and the analysis is an important factor. This acknowledges the fact that calculations however detailed and sophisticated will not necessarily come up with the correct predictions due to various unknowns in assessment procedure. These unknowns can be attributed to a number of factors, many of which are beyond the control of the engineer using the code. They are as follows:

- The available material property data for the analysis are invariably insufficient or crude and since they are usually taken from historical data, results from different batches of material or tests in different laboratories with insufficient number of test specimens, are likely to contain a large scatter.

- The scatter and sensitivity in creep properties inherently produce a large variation in the calculations. Upper and lower bounds are therefore introduced which give widely different life prediction results.

- The evaluations of the relevant parameters such as $K$, limit load concepts, reference stress $\sigma_{r e f}$ and $C^{*}$ are different according to the method of derivation.

- The use of short-term small laboratory data for use in long term component life predictions further increases the possibilities of a wrong prediction.

- Difficulty in ascertaining the level of crack tip constraint and multi-axiality effects in the component will reduce the accuracy of crack growth predictions by about a factor of 30 .

- Unknowns in modelling the actual loading history, component system stresses and additional unknowns such as little or no knowledge of past service history, residual stresses etc., act as sources of error in predictions.

- Limitations of non-destructive (NDT) methods for measuring defects in components, during operation and/or shutdown and insufficient crack measurement data during operation, are likely to add to the errors.

All these factors suggest that however detailed sophisticated and accurate a particular calculation is the results will still need to be treated with caution. In addition the similarity of the approaches in the various codes do not necessarily imply that calculations by the different methods will give the same predictions. It 
may be possible that under certain controlled and validated circumstance, such as the A16 evaluation for the $316 \mathrm{LN}$ stainless steels used in the fast reactor industry, the predictions can be optimised. In this latter case the detailed model of the creep behaviour of $316 \mathrm{LN}$ steel is used for the analysis [11]. It is clear that a critical comparison is only possible when the same method is used on another material and condition or the same test cases are examined by the different codes.

To deal with these unknowns in the life assessment all codes employ safety factors to a greater or lesser extent though the origin of these is not always admitted. These factors are based on bounds of experimental data and the levels of accuracy that the parameters can be calculated to. Another approach is to use statistical methods to produce upper and lower bounds of the data and the parameters. But if proper judgement is not used in such cases, the life estimations could become extremely conservative and hence possibly useless for both design and operational purposes. Therefore both qualitative as well as quantitative judgement is needed to make a meaningful life assessment.

\section{Loading and stress analysis}

The principles of stress definition in creep and fatigue crack growth are similar between the codes. The procedures define primary stresses in tension and bending (which are not relaxed in operation), secondary stresses (which are relaxed) and peak stresses (which are local and self equilibrating). The relevant nominal stress is input into the codes for evaluating the fracture mechanics parameters $K, J, \sigma_{r e f}$ and $C^{*}$. Solutions for $K$ and $J$ are available in the literature for most standard component shapes. For more complicated structures finite element analysis is used. Limit load analysis methods are used to determine $\sigma_{\text {ref }}$ using

$$
\sigma_{r e f}=\sigma_{y} \frac{P}{P_{l c}}
$$

where $P_{l c}$ is the collapse load of a cracked body and $\sigma_{y}$ is the yield stress. The value of $P_{L C}$ will depend on the collapse mechanism assumed and whether plane stress or plane strain conditions apply. The $\sigma_{r e f}$ is the used to calculate $C^{*}$ in components using [2]

$$
C^{*}=\sigma_{\text {ref }} \dot{\varepsilon}_{\text {ref }}\left(\frac{K}{\sigma_{\text {ref }}}\right)^{2}
$$

where $\dot{\varepsilon}_{\text {ref }}$ is the creep strain rate at the reference stress. In this case numerical, analytical and experimental information can be employed to evaluate the parameter $C^{*}$. The R5 and A16 procedure also contain compendia of the fracture mechanics parameters for relevant cracked geometries and suggests that the main parameters can be calculated using appropriate finite element analysis. The FBH approach for stress estimation is in accordance with the German standards (TRD, KTA) and also the ASME pressure vessel and piping code Section III.

The reference stress approach [2] is based on the concept that deformation in a structure can follow the same route as deformation in a uniaxial test at an appropriately chosen stress defined as the reference stress. Its simplicity is based on the fact that detailed finite element calculations are not needed since it uses the limit load analysis concept to calculate the reference stresses. However the solutions for $C^{*}$ using equation (2) which use $K$ and, more so, $\sigma_{\text {ref }}$ are a substantial source of difference which determines the outcome of the defect assessment results [12], since different methods of evaluations will yield widely 
different results. A different solution is used to calculate $\sigma_{\text {ref }}$ in each procedure depending on the yield criteria employed.

For standard laboratory testing [12-14] where fracture mechanics specimens are employed to produce material crack growth properties at elevated temperatures, $C^{*}$ is derived experimentally using the loadline creep displacement rate [13] giving

$$
C^{*}=\frac{P \dot{\Delta}}{B_{n} W} F
$$

for a specimen of thickness $B_{n}$ between side-grooves, width $W$ and subjected to a load $P$. In this expression $\dot{\Delta}$ is the experimentally measured load point creep displacement rate and $F$ is a nondimensional factor which depends on the stress sensitivity of creep $n$ and crack length $a$ [12-14]. Most data bases for experimental creep crack growth use equation (3) for analysis but without proper validation between the two methods there may exist a clear discrepancy between results obtained from equation (2) and equation (3).

The evaluation of $\dot{\Delta}, \dot{\varepsilon}_{f}$ used in eqns. (20 and (3) which are derived taken from experimental load displacement, stress/strain rate or stress/rupture plots is also factors which will determine the accuracy of life assessment results. Finally both creep uniaxial and creep crack growth data will inherently contain experimental scatter bands that will need to be considered in a life assessment procedure [15].

\section{A unified method for life assessment}

From the review of the present codes it emerges that life predictions based only on purely procedural and deterministic calculations specified by the codes will not necessarily give correct answers. It is important that a new procedure should adopt a comprehensive and open approach by adopting different methodologies for the prediction of creep crack initiation and growth based on both deterministic and probabilistic techniques. It is also important that the new procedure would have to be more dependent on a knowledge base and user experience approach. In such a case the information and relevant advice would 'build up' over time from experience gained in either previous analyses or from new tests and databases or direct from plant experience. The computer programme R5-code based on R5 has moved towards such a methodology. This type of 'sensitivity' analysis can be conveniently developed using inter-active computational methods. The different stages of calculations would be as follows;

\footnotetext{
Stage 1) Input $1 \quad$ Problem specification - design or life assessment

Stage 2) Input 2 Material properties/details from database or other sources- actual /mean/upper and lower bounds data.

Stage 3) Input 3 Geometries: Laboratory test/feature tests/components.-crack shape, size

Stage 4) Input 4 System stresses/loading/loading history/residual stresses- actual/mean/upper and lower bounds data.

Stage 5) Output Calculation of $K / \Delta K / J / \sigma_{r e f} / C^{*}$ at different crack lengths - upper/lower bounds depending on the evaluation method

Stage 6) Checks - Static check for fast fracture and collapse

Stage 7) Checks - Ligament rupture creep analysis

Stage 8) Analysis 1 Creep or creep/fatigue initiation

Stage 9) Analysis 2 Fatigue crack growth
} 
Stage 10) Analysis 3 Creep crack growth

Stage 11) Analysis 4 Creep/fatigue interaction

Stage 12) Analysis 5 Sensitivity analysis/comparisons//probabilistic analysis

These stages can be developed in detail to deal with different material as well as loading and structural variations. They are further described as follows

\section{Initial material information - Stages 1-4}

Stages 1-4 are the input of information stage where it is important to remind the user of the bounds and the accuracy of the data that will be employed in the calculation stage. In remnant life assessment procedures the emphasis, where material properties are concerned, should be based on five areas. These are as follows;

- Building up a comprehensive materials database from the test program to complement the existing information available.

- Interpretation of data from feature and component tests if available.

- Interpretation of historical data from the actual component if available.

- Acquiring the relevant material properties needed for the modelling stage.

- Detailed knowledge of service history

\section{Calculations -Stage 5}

The relevant nominal stress is evaluated using information about the primary stresses in tension and bending, secondary stresses, and peak stresses. Loading and temperature history need to be taken into account as well. This information is used for evaluating the right nominal stresses relevant to calculating the fracture mechanics parameters $K, J, \sigma_{\text {ref }}$. Equations (1) and (2) are used for the calculation of $C^{*}$. In this stage as there is no universally accepted fool proof method of determining the parameters it is essential that the user is given bounds and different methods of calculating the parameters, where possible. The available methods for evaluating the relevant fracture mechanics parameters should also provide levels of sensitivity of these parameters with respect to the final calculations. This information can be built up when validations are performed for a particular situation.

\section{Pre-analysis checks -Stages 6-7}

The pre-analysis stage is where the structure is checked against catastrophic ligament fracture and plastic and creep collapse. These stages check incrementally the status of the structure with respect to collapse and creep failure. Universally acceptable methods [2] are available to deal with these stages of calculation. However the checks made and the limits advised are dependent on the accuracy of the material properties and the methods of calculating $K$ and $\sigma_{\text {ref. }}$ Following this stage, stages 8-11 deal with the crack initiation and development of crack growth in a creeping material under static and cyclic loading conditions.

\section{Modelling Initiation - Stage 8}

The methods of analysis for initiation are outlined below. The variables needed should have all been checked and completed in the previous stages. The basic assumption in the analysis is the presence of a defect and the extension of it due to creep and/or fatigue. Stage 8 assumes that the initial part of the life of the component is taken up by damage development where the crack does not extend beyond a predetermined length. The length is taken from either the accuracy limits of NDT measurements or set by a standard (usually chosen in the range of $100-500 \mu \mathrm{m}$ ). For purely laboratory testing ASTM E1457 
recommends $200 \mu \mathrm{m}$, which is found to be within the steady state cracking [12] region for most engineering materials and loading conditions.

As shown in Figure 1 this initiation region could be a substantial period of life and as a result of the flat slope followed by a sharp crack extension, it is relatively insensitive to crack length. The present codes deal with this region with widely different methods. It is usual for the codes to discount initiation when fatigue is present. The methods proposed have been successfully employed in different European assessment codes[1-9]. Since little universal validation exist to justify the choice of one method over another it is proposed to implement four models for crack initiation at this stage. It should be noted that as experience and validation and modelling techniques develop, for a particular methodology and material, then this information can be included as advice to the user eg. As a suggestion for the preferred choice of analysis for a particular situation. The different models proposed are as follows;

\section{Method 1: Sigma $\sigma_{D}$ approach}

The principle is based on calculating damage accumulation by creep and fatigue in a cracked structure [4-5] and this method is an extension of the same methodology for un-cracked structures with the exception that a specific stress $\sigma_{D}$ is defined at a specific distance from the crack tip. The method to determine strain ranges $\Delta \varepsilon_{i}$, the stress $\sigma_{i}$ (accounts for elastic + plastic strains +creep strains) at the distance $D$, which is material dependent and usually taken as $50 \mu \mathrm{m}$, from the crack tip. Fatigue damage is determined by calculating strain ranges for all cycles in the life $N_{\mathrm{L}}$. Number of cycles to failure at each strain range is determined from fatigue endurance data.

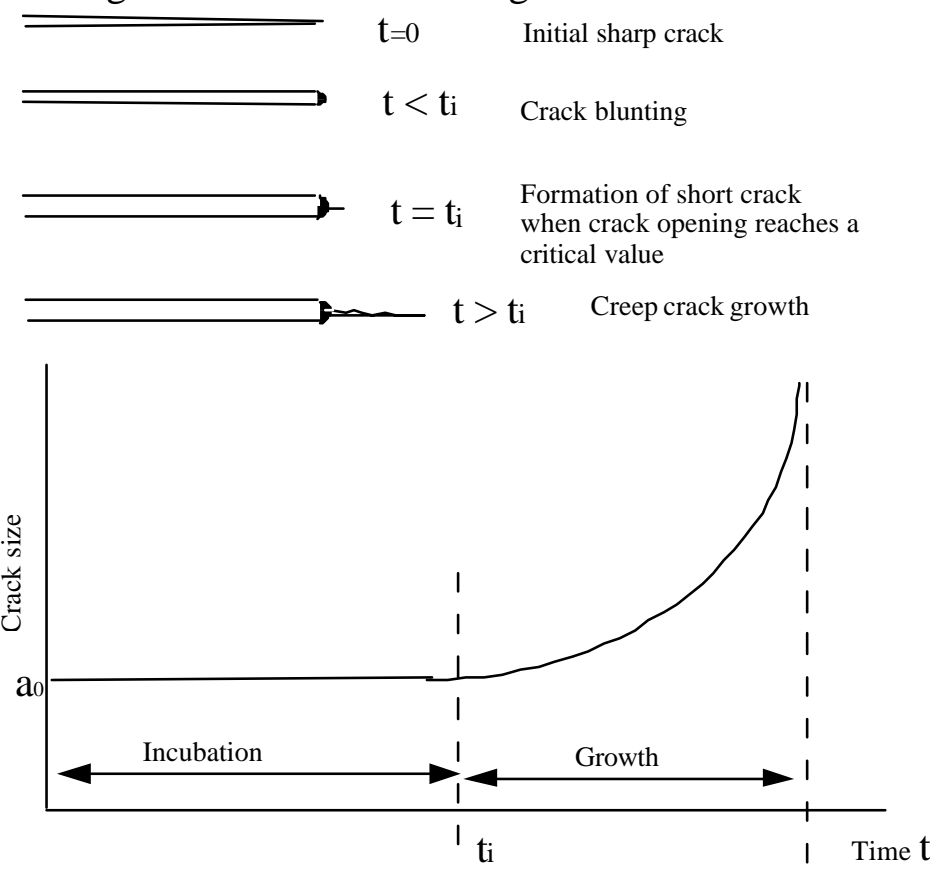

Figure 1: Schematic behaviour of a crack at elevated temperature

Finally all ratios of the numbers of cycles experienced at each strain range over the maximum number of cycles at that strain range are summed. Calculation of the creep damage is the same as for fatigue but damage calculation includes elastic, plastic, and creep strains. Creep damage is determined for all cycles and rupture time used up is determined from the fraction as the sum over all ratios of numbers of cycles. If the fatigue and creep fraction points $\left(D_{\text {fat }}, D_{\text {cr }}\right)$ lie within the fatigue-creep interaction diagram no 
initiation occurs as shown in Figure 2. If initiation did occur then the initiation time $t_{\mathrm{I}}$ is determined through an iterative procedure. The last point at the intersection boundary to initiation determines the timed cycles to initiation.

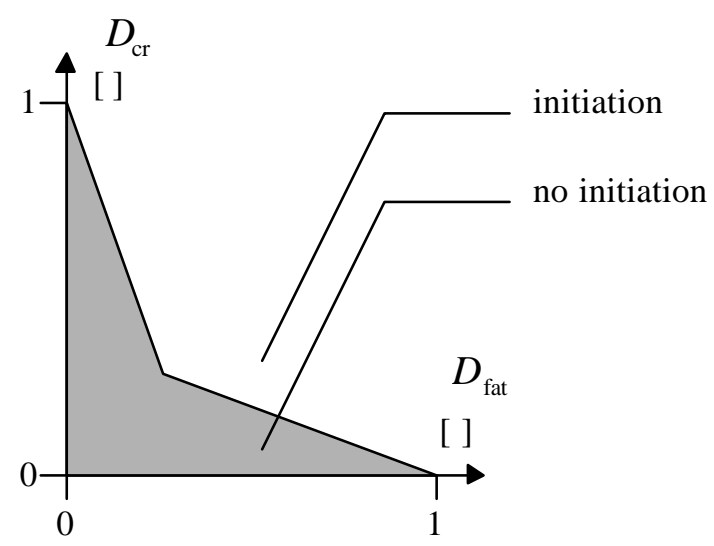

Figure 2: Example of a fatigue-creep interaction diagram

\section{Method 2: The $\delta_{I} / C *$ approach for initiation}

In this method non-linear fracture mechanics based modelling is employed to analyse the initiation period of a small crack extension [1-3]. This method uses the reference stress method for evaluating $C^{*}$ and assumes that the crack tip stresses can be described by steady state $C^{*}$. If experimental data is available the initiation time $t_{i}$ is calculated

$$
\begin{aligned}
& R^{\prime}=\left(\frac{K}{\sigma_{\text {ref }}}\right)^{2} \\
& t_{\mathrm{I}}=\left[\frac{\left(\delta_{\mathrm{I}} / R^{\prime}\right)^{n /(n+1)}-\sigma_{\mathrm{ref}} / E}{D \sigma_{\mathrm{ref}}^{n}}\right]^{1 / p}
\end{aligned}
$$

Where $\delta_{\mathrm{I}}$ is the crack opening displacement at initiation (material dependent), $E$ is the Young's modulus and $D, n, p$ are creep material properties. Incubation time is measurable from test specimens and comparing the experimental results from the specimen with the component gives

$$
\left(\frac{t_{\text {Icomp }}}{t_{\text {Ispec }}}\right)=\left(\frac{C_{\text {spec }}^{*}}{C_{\text {comp }}^{*}}\right)^{n /(n+1)}
$$

where $t_{\text {Icomp }}$ is the unknown time to initiation of the component, $t_{\text {Ispec }}$ is the known time to initiation of the specimen, $C^{*}{ }_{\text {spec }}$ is known parameter defining creep crack propagation rate of the specimen and $C^{*}$ comp is known parameter defining creep crack propagation rate of the component. If fatigue is present then initiation time is taken as zero.

\section{Method 3: Two criteria approach}

This method [7,8] is only relevant to creep initiation and does not consider fatigue. Initiation is given if the point lies in the specific two criteria dagram, which depends on material or at least material group properties. It compares ligament damage against crack tip damage (Figure 3) 


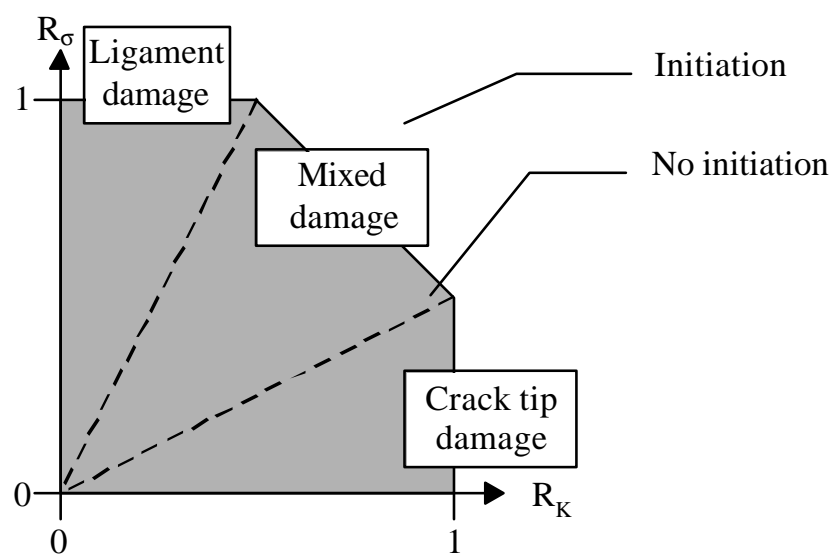

Figure 3: Example of the two-criteria crack initiation diagram

Where, $R_{\mathrm{K}}=\frac{K}{K_{\mathrm{I}}}$ and $R_{\sigma}=\frac{\sigma_{\mathrm{no}}}{R_{\mathrm{mt}}}$

and where $\sigma_{\text {no }}$ is the nominal stress in the far-field of a pre-cracked component (fully redistributed stresses), $R_{\mathrm{mt}}$ is the creep rupture strength of a smooth tensile specimen (function of time, e.g. taken at $\left.10^{4} \mathrm{~h}\right), K$ is elastic $K$-value at the crack tip of the component and $K_{\mathrm{I}}$ is $K$-value at creep crack initiation experimentally determined as a function of time, (e.g. taken at $10^{4} \mathrm{~h}$ ). If fatigue is present then initiation time is taken as zero.

\section{Method 4: $C^{*}$ Transient method}

This method also uses fracture mechanics principles to determine the time taken for creep damage to accumulate at the crack tip starting from first initial elastic loading [12,16]. Essentially the steady state creep crack growth rate $\dot{a}$ can be correlated satisfactorily in terms of $C^{*}$ by the relation

$$
\dot{a}=D_{\mathrm{o}} C * \phi
$$

where $D_{\mathrm{o}}$ and $\phi$ are material constants which can be measured experimentally using the NSW model [14] based on a creep zone model. Equation (7) can be approximated to

$$
\dot{a}_{s}=\frac{3 C^{* 0.85}}{\varepsilon_{f}^{*}}
$$

where $\dot{a}_{s}$ is the steady crack growth rate in $\mathrm{mm} / \mathrm{h}, \varepsilon_{f}^{*}$ is failure strain as a fraction and $C^{*}$ is in $\mathrm{MJ} / \mathrm{m}^{2} \mathrm{~h}$. The relevant ductility needed for crack growth is $\varepsilon_{f}^{*}=\varepsilon_{f}$ (the uniaxial ductility) and it could be reduced in the limit by a further factor of $\varepsilon_{f}^{*}=\varepsilon_{f} / 30$ in the plane strain regime. This range describes the effects of constraint on crack growth due to both material properties and size/geometric factors.

The initial stage of cracking prior to steady growth exhibits a transient phenomenon [12-16] due to the build up of damage at a crack tip prior to the onset of steady state behaviour. This can lead to an incubation (as shown in Fig. 1) period before measurable crack growth can be detected [16]. If the minimum crack extension that can be resolved reliably is $\Delta a$ then the incubation period $t_{\mathrm{i}}$ is given by 


$$
t_{\mathrm{i}}=\int_{0}^{\Delta a} \frac{d r}{\dot{a}}
$$

where $d r$ is distance from the crack tip. Bounds on $t_{i}$ can be obtained by representing cracking rate by its initial value $\dot{a}_{o}$ and its steady state value $\dot{a}_{s}$. The initial cracking rate can be approximated to the steady state rate in eqn. (7) by

$$
\dot{a}_{0}=\dot{a}_{s} /(n+1)
$$

The value of $n$ for most engineering materials is usually in the range 5-10, which suggest that $\dot{a}_{o}$ is approximately an order of magnitude less than its steady state value. Thus eqn. (7) for steady state conditions gives a lower bound incubation period of

$$
t_{\mathrm{iL}}=\Delta a / D C^{* \phi}
$$

or using the approximate eqn. (8)

$$
t_{i L}=\frac{\Delta a \varepsilon_{f}^{*}}{3 C^{* 0.85}}
$$

Alternatively if the incubation period is calculated from the initial transient cracking rate $\dot{a}_{o}$ determined from eqn. (10) the approximate upper-bound $t_{i U}$ to the initiation time becomes

$$
t_{i U}=\frac{(n+1) \Delta a \varepsilon_{f}^{*}}{3 C^{* 0.85}}
$$

In eqn. (11-13) the incubation period is proportional to $\Delta a$. The limit of reliable crack detection is at best $\pm 100 \mu \mathrm{m}$ (which is the level set for standard CT testing in ASTM E1457-98) and can sometimes be as large as $0.5 \mathrm{~mm}$. This range is examined in the investigation.

\section{Modelling creep and creep/fatigue crack growth -Stages 9-11}

As this paper is exclusively concerned with crack initiation and crack growth procedures the following sections will deal mainly with comparing the different codes with respect to their methodologies of dealing with the problem of creep crack initiation and growth under static and cyclic loading. The relationship given in equation (7) for crack growth predictions is the same in all codes.

At room temperature under cyclic loading conditions, crack propagation usually occurs by a fatigue mechanism where the Paris Law can describe crack growth/cycle $(d a / d N)_{\mathrm{F}}$ in terms of stress intensity factor range $\Delta K$ by

$$
(d a / d N)_{\mathrm{F}}=C \Delta K^{m}
$$

Where $C$ and $m$ are material dependent parameters, which may be sensitive to the minimum to maximum load ratio $R$ of the cycle. At elevated temperatures combined creep and fatigue crack growth may take 
place. Previous studies [17] have shown that a simple cumulative damage law can be employed to describe this behaviour. The law states that the total crack growth/cycle $(d a / d N)$ can be obtained from

$$
\left(\frac{d a}{d N}\right)=C \Delta K^{m}+\frac{\dot{a}}{f}
$$

where $f$ is frequency and $\dot{a}$ is the creep component of cracking which can be determined from equation (7) or any of the previous models of creep crack growth [12]. Therefore by determining the crack growth rate according to eqn. (7) eqn. (15) becomes

$$
\left(\frac{d a}{d N}\right)_{c}=\int_{0}^{t_{h}} \dot{a}\left[C^{*}(a)\right] d t
$$

where the value of the parameter $C^{*}$ can be taken at the start of the holdtime as an upper bound but expressions are available for its determination under displacement-controlled conditions. The two contributions in equation (15) are summed over the operating cycles, updating the crack size as necessary. This linear cumulative damage law has been used in the analysis and assumes little or no interaction between the time dependent creep and the time independent fatigue component of cracking.

\section{Review of results and sensitivity analysis -Stage 12}

Comparisons, checks and advice for the user should be included at every stage. This stage is termed as the sensitivity analysis stage where the user is urged to evaluate the results by varying the input and the method of calculations in stages 1-5. The use of mean/upper and lower bounds will identify for the user the sensitivity of the predictions. The implementation of probabilistic assessment methods [19-20] is an option that the KBS should implement. The KBS in the HIDA project[see acknowledgement] is written in such a way so as to allow flexibility in implementing additional 'modules' in order to extend the analysis and prediction capabilities of the KBS for the future. In particular when probabilistic methods are developed the KBS should be able to be adapted to deal with the new calculations.

\section{Conclusions}

It has been shown that there are many similarities between the various European codes, which deal with defects in components. The German flat bottom hole approach (FBH) concentrates upon crack detection and characterisation as an important factor in the life assessment. The German two criteria method considers only initiation of a crack for life assessment. The more sophisticated approaches of the French A16, the British Energy's R5 and BS-7910 crack initiation and growth assessment codes have been developed based on clear similarities. However their range of validation based on long-term data is good for specific usage but is limited in most other circumstances.

The use and the evaluation of the relevant fracture mechanics parameters need to be reviewed and the ranges of their applicability more clearly defined. The use of different techniques of modelling and assessing a problem is important, as it will act as a sensitivity analysis for the predictions. In addition when a defect is discovered or where a hypothetical defect is assumed, the codes should be able to check the flaw sensitivity of a proposed design and it should be possible to benefit from the initiation/incubation period before the crack starts to grow. The consistent way of critically comparing approaches is to implement a comprehensive sensitivity analysis at the calculation stages, which will give the user additional confidence in the accuracy of the calculations. 
Since most codes have been developed as a result of experience gained from material specific programs it is suggested that improvements to the codes can be made by simplification of the methodology [18]. It will also allow the codes to be more applicable to a wider range of materials and geometries. The approach in life assessment should to set confidence limits on the predictions based on deterministic and probabilistic methods. Finally as assessment codes are invariably applied at plant level the simplification for their use can best be accomplished by using a computerised Knowledge Based System [21] in which the user is advised at every stage of the procedure and is given the opportunity for comparing the analyses.

\section{Acknowledgements}

The author would like to thank the European Commission for their financial contribution and the Partners in the 'HIDA'-High-Temperature Defect Assessment project: ERA Technology (UK); Imperial College (UK); MPA Stuttgart (Germany); Siempelkamp (Germany); CEA (France); EDF (France); SAQ Kontroll/SIMR (Sweden); ENEL (Italy); Petrogal/ISQ (Portugal); Metsearch (Netherlands); Framatome (France) for the provision of data.

\section{References}

1) Goodall, I. W. (ed.) (1990) Assessment procedure for the high temperature response of structures, Nuclear Electric procedure R5, Issue 1.

2) Ainsworth, R. A. and Budden, P. J. (1994) Design and assessment of components subjected to creep, J. Strain Anal., 29, 201-208.

3) Ainsworth, R. A., Chell, G. G., Coleman, M. C., Goodall, I. W., Gooch, D. J., Haigh, J. R., Kimmins, S. T. and Neate, G. J. (1987) CEGB assessment procedure for defects in plant operating in the creep range, Fatigue Fract. Eng. Mater. Struct. 10, 115-127.

4) Drubay, B., Moulin, D., Faidy, C., Poette, C. and Bhandari, S. (1993) Defect assessment procedure: a French approach, ASME PVP, Vol. 266, 113-118.

5) Green, D., Moulin, D. and Hooton, D. (1995) Development of fracture assessment procedures at elevated temperature in Europe, in SISSI 94, Saclay International Seminar on Structural Integrity: Principles of Fracture Mechanics Applications in Nuclear Power Plants, Centre d'Etudes de Saclay, pp. 59-71.

6) British Standards BS $7910: 1999$, Guide to methods of assessing the acceptability of flaws in fusion welded structures, BSI, London, 1999.

7) Ewalds, H. L. \& Wanhill, R.J.H., Fracture Mechanics, Edward Arnold, 1985.

8) Ewald, J. \& Keienburg, K-H, 'A two criteria diagram for creep crack initiation', Int. Conf. Creep, Tokyo, April 1986, 173-178.

9) ASME Boiler and Pressure Vessel Code (1991), Case N-47 (29), Class 1 components in elevated temperature service, Section III, Division I, ASME, New York.

10) RCC-MR (1985) Technical Appendix A3, Section 1, Subsection Z, Materials design and construction rules for mechanical components of FBR nuclear test islands, AFCEN, Paris.

11) Poussard, C. \& Moulin, D., (1998), 'Creep-Fatigue Crack Growth in Austenitic Stainless Steel Centre Cracked Plates at $650{ }^{\circ} \mathrm{C}$ - Part I: Experimental Study and Interpretation', Journal of Materials at High Temperature, 15(3/4), 313-330, 1998.

12) G. A. Webster, K. Nikbin, M. R Chorlton, N.J.C. Celard, M. Ober, 'A comparison of High temperature defect assessment methods', Journal of Materials at High Temperature, 15(3/4) , pp.337-347, 1998. 
13) ASTM (1998) 'Standard test method for measurement of creep crack growth rates in metals', ASTM E1457:1998, Vol. 03.01, 1031-1043.

14) Nikbin, K. M., Smith, D. J., and Webster, G. A., "An Engineering Approach to the Prediction of Creep Crack Growth," Journal of Engineering Materials and Technology, Trans. ASME, Vol 108, 1986, pp. 186-191.

15) Nikbin, K., 'Consideration of safety factors in the life extension modeling of components operating at high temperatures' in 'Effects of product quality control and design criteria on structural Integrity', ASTM STP 1337, Eds. R. C. Rice, D. E. Tritsch, American Society for Testing and Materials, p. 20-33, (1998).

16) Kaguchi, H., Djavanroodi, F., Nikbin, K. M., Webster, G. A., 'Prediction of transient effects during the early stages of creep crack growth', Ed. K.T. Rie, published in the proceedings of the DVM Conference, Berlin, Sept. (1994).

17) Winstone, M.R., Nikbin, K. M. And Webster, G.A., 'Modes of failure under creep/fatigue loading of a nickel-based superalloy', J. Mat. Sci., 20, 1985, 2471-2476.

18) Penny, R. K., 'Robust methods for Plant life assessments', R. K. Penny and Associates, ASME Conference on Pressure Vessels and Piping, Orlando, Fl., 1997, p1-7.

19) Cane, B. J. and Browne, R. J.,' Risk-based inspection - the needs, benefits and approach for power process plants', in 'Life management and evaluation of plant, structures and composites', Eds. J. H. Edwards, P. E. Flewitt, B. C. Gasper, P. Stanley and B. Tomkins, EMAS Publishers., pp-461-476, 1988.

20) Millwater, H. R., Fitch, S. K. H., Justin, Y. -T., Riha, D. S., Enright, M. P., Leverant, G. R. McClung, R. C., Kuhlman, C. J., Chell, G. G. and Lee, Y-D, ' A Probabilistically based damage tolerance analysis computer program for hard alpha anomalies in titanium rotors', Presented at the Int. Gas Turbine and Aeroengine Congress, Munich, Germany- May8-11 , 2000.

21) Jovanovic, A.S. and Wagemann, G., 'Knowledge-Based System (KBS) for creep crack growth of high temperature components in HIDA project', Journal of Materials at High Temperature, 15(3/4), pp347-355, 1998. 